FORMATION Formation emploi

Revue française de sciences sociales

108 | octobre-décembre 2009

Les processus de professionnalisation

\title{
La quête de professionnalisation des communicateurs publics : entre difficulté et stratégie
}

The search for skills training for public communicators: between difficulty and strategy

Streben der Berufe der öffentlichen Kommunikation nach Professionalisierung:

Zwischen Schwierigkeit und Strategie

La busqueda de profesionalizacion de los comunicadores publicos : entre

dificultad y estrategia

Dominique Bessières

\section{OpenEdition}

\section{Journals}

Édition électronique

URL : http://journals.openedition.org/formationemploi/2081

DOI : 10.4000/formationemploi.2081

ISSN : 2107-0946

Éditeur

La Documentation française

Édition imprimée

Date de publication : 1 décembre 2009

Pagination : 39-52

ISSN : 0759-6340

Référence électronique

Dominique Bessières, « La quête de professionnalisation des communicateurs publics : entre difficulté et stratégie », Formation emploi [En ligne], 108 | octobre-décembre 2009, mis en ligne le 01 janvier 2012, consulté le 30 octobre 2020. URL : http://journals.openedition.org/formationemploi/2081 ; DOI : https://doi.org/10.4000/formationemploi.2081 


\section{DOSSIER}

\section{La quête de professionnalisation des communicateurs publics: entre difficulté et stratégiel}

Par Dominique Bessières*

Les communicateurs publics, souvent contractuels, peinent à intégrer le modèle dominant du fonctionnaire recruté par concours; dès lors, ils recherchent des stratégies alternatives de professionnalisation.

Les revendications de distinction en groupe professionnel visent à obtenir la reconnaissance d'un professionnalisme particulier. C'est ce que nous souhaitons étudier auprès des communicateurs publics ${ }^{2}$, principalement auprès des responsables de communication institutionnelle. Ils ont pour charge de gérer les services de communication et souvent l'intervention d'agences prestataires. Ils définissent les plans et les actions de communication: relations publiques et presse, site internet, magazine institutionnel, affichage, plaquettes, évènement... Leur généralisation dans les organisations publiques est assez récente (Bessières, 1998, pp. 134-147). Des signes de leur professionnalisation et de leur institutionnalisation montrent une recherche de stabilisation.

\footnotetext{
${ }^{1}$ L'article résulte du développement d'analyses post thèse de doctorat sous l'angle de la sociologie des professions, avec plusieurs vagues et terrains.

${ }^{2}$ Constitutifs d'une branche particulière très peu analysée de la sociologie des professions de cadre (Gadéa 2003, p. 212). On trouve aussi l'appellation de communicant public, directeur ou chargé de communication, dircom...
}

Le modèle professionnel dominant dans le secteur public est celui des fonctionnaires recrutés par voie de concours, ce qui contribue à disqualifier les agents

* Dominique Bessières est maître de conférences à I'IUT (Institut universitaire de technologie)/ Laboratoire d'étude et de recherche sur les professionnalisations, université de Reims Champagne-Ardenne / Institut d'études politiques de Lille, 7le section «sciences de l'information et de la communication ».

Domaines de recherche : Professionnalisation de la communication publique, Technologies de l'information et de la communication de l'enseignement, Risques environnementaux et communication des institutions publiques.

Il a publié récemment : " Le modèle des professions, enjeu de légitimation étatique pour la communication publique simulacre ou effet de réalité », Colloque international, ISA International Sociological Association / Association Internationale de Sociologie, 5e Conférence intermédiaire du Comité de recherche 52 "Sociologie des groupes professionnels", Le modèle des professions en question : apports et limites du professionnalisme, Oslo University College, Norvège, 12-13 septembre 2008 
recrutés différemment. Leur carrière est organisée dans le respect des prescriptions du droit administratif de la fonction publique, avec des variations entre les fonctions publiques étatique, territoriale ou hospitalière ${ }^{3}$. Or, dans la fonction publique, les responsables de la communication sont majoritairement contractuels ; ceci les conduisant à se percevoir minoritaires par rapport à leur représentation des autres cadres dirigeants titulaires de la fonction publique ${ }^{4}$, ils recherchent des stratégies alternatives de professionnalisation calquées sur celles du secteur privé, afin de conforter leur position minoritaire encore fragile. De telles revendications se renforcent continuellement depuis les années 80. La décennie 1975-1985 étant caractérisée par des profils politisés de militants des

${ }^{3}$ Notre enquête ne traite par de la FPH (fonction publique hospitalière).

${ }^{4}$ L'ensemble de nos entretiens confirme ce ressenti. équipes chargées de la communication au niveau local, la décennie suivante par le recrutement de personnels spécialisés (Pailliart 1993, pp. 92-95).

Leur professionnalisation, dans sa dynamique, est ambivalente et donc problématique dans la mesure où elle emprunte aux deux références publique/privée. Plus largement, elle est polysémique et ne se réduit pas à une seule acception. Elle peut viser la constitution d'une nouvelle profession, une socialisation socle d'un développement de carrière, la formation de professionnels par des instances d'enseignement ou encore un processus de légitimation de la spécialisation des fonctions professionnelles de communication atypiques dans le secteur public ; en ce sens, elle est un facteur d'identité professionnelle (Dubar, Tripier). Ce processus toujours en construction s'inscrit dans la durée, avec une conjonction de facteurs et d'échelles sociologiques différentes.

\section{Encadré 1 \\ Méthodologie}

Notre méthodologie est celle de la «boite à outil » constituée d'un ensemble ad hoc d'observations, de méthodes, de théories (Thoenig 1985, p. 4)

Elle repose sur la compilation de 68 entretiens semi-directifs (dont 11 femmes) diachroniques d'acteurs du champ issus de différents terrains : responsables de communication, d'associations ou de salons professionnels, concepteurs de référentiels de compétences. Ils se sont déroulés entre 1993 et 2008, d'une durée de 40 minutes pour les plus brefs à deux heures pour les plus longs, voire trois heures dans quelques cas impliquant plusieurs entretiens. Ce corpus intègre plusieurs vagues et terrains, avec des questionnements communs sur les actions de communication menées, leurs contextes organisationnels spécifiques, leurs représentations par rapport à leur rôle.

33 entretiens auprès des services de communication des collectivités locales des départements et de la région francilienne (1993-1997).

6 entretiens de responsable de communication champ'ardennais (Conseil régional, agglomération, $\mathrm{CCl}$ - chambre de commerce et d'industrie -, agence publique de développement économique, 20062008).

5 responsables de communication d'administration centrale (2006-2008).

4 directeurs de la communication de villes importantes de province (2006-2008).

6 entretiens de responsables d'associations professionnelles (1994, 2008).

11 entretiens de conseils en communication (1993-1997).

3 journalistes spécialisés (1993-1997).

L'analyse s'appuie également sur des examens documentaires de discours performatifs (lois, code de déontologie).

Enfin, elle intègre des éléments d'observation de manifestations organisées par des groupements d'acteurs depuis plus de 15 ans. 
Dans ce contexte, il est frappant de constater que les critères classiques des professions ${ }^{5}$ paraissent scrupuleusement observés et mis en pratique pour offrir une image proche et décalquée des modèles reconnus en la matière. Dubar et Tripier (2005, pp. 5-7) ont établi quatre sens au concept de profession que nous aborderons: une identité professionnelle (déclaration), une spécialisation professionnelle (métier), une position professionnelle (fonction), une classification professionnelle (emploi). La construction collective de la reconnaissance de la fonction de communication est métissée entre culture professionnelle et organisations publiques; elle se manifeste par des ajouts successifs. Aussi, dans quelle mesure les modes de construction et de légitimation en groupe professionnel des communicateurs publics constituent-ils une condition d'intégration pérenne dans une structure de travail fonctionnarisée ?

Nous verrons le particularisme des communicateurs publics à la recherche de distinction du groupe, sa consolidation par des acteurs de sa représentation, enfin le rôle de reconnaissance de l'État dans la société.

\section{DES CONTRACTUELS DANS DES ORGANISATIONS FONCTIONNARISÉES}

La fonction publique française se caractérise historiquement par la prégnance des concours administratifs portant sur des matières disciplinaires censées garantir des agents publics de qualité, dans le droit fil du modèle bureaucratique wébérien, avec une neutralité des fonctionnaires à l'égard des pouvoirs politiques, et une certaine forme de protection vis-à-vis de pressions pouvant émaner de ces derniers. À ce titre, le concours constitue la voie normale d'accès à la fonction publique. Dans ce contexte, la fonction de communicateur public a progressivement émergé. Juridiquement, le recrutement de contractuels peut être opéré lorsqu'il n'existe pas de corps de fonction-

\footnotetext{
${ }^{5}$ Nous utilisons le mot profession dans un sens proche de l'anglais « occupation », mais avec des éléments inspirés des professions établies (proche du sens anglais de «profession »).
}

naires correspondant, ou pour des emplois de catégorie A (dits de « cabinet ») si la nature des fonctions ou les besoins du service le justifient ${ }^{6}$ : ces modalités spécifiques et contingentes peuvent occasionner des difficultés de visibilité, voire de transparence, en particulier dans leur dénombrement.

\section{Encadré 2 Le chiffrage des contractuels}

Les contractuels peuvent être classés dans la rubrique "cadre» de la fonction publique (nomenclature Insee). Pourtant, leur chiffrage n'est pas aisé avec différents niveaux statutaires: les agents non-titulaires sur emploi non permanent, et permanent depuis 2005 (contractuels, vacataires, auxiliaires). Aussi le ministre Woerth, en présentant l'agenda social 2009 pour la fonction publique, pointe la nécessité d'un état des lieux de la situation des agents contractuels dans la fonction publique (Conseil des ministres du 11 mars 20091.

Toutefois, en 2006, officiellement la part des non-titulaires dans la fonction publique est minoritaire, soit $14,9 \%$ dans les trois fonctions publiques contre $74,4 \%$ de titulaires lauxquels s'ajoutent les militaires et volontaires militaires, les médecins, les assistantes maternelles et les ouvriers d'État... : http:// www.fonction-publique.gouv.fr/IMG/ Chiffres_cles_2008.pdf I et est globalement en diminution entre 2005 et 2006 (http://www.acteurspublics.com/article/ 12-09-08/moins-de-contractuels) à l'exception de la fonction publique territoriale $(+6,5 \%)$ avec un agent contractuel sur cinq. Mais dans un autre chiffrage, les contractuels représenteraient $41 \%$ des emplois permanents à temps complet dans les fonctions administratives en 2001 (ADF- Bilans sociaux des conseils généraux au $31 / 12 / 011$.

\footnotetext{
${ }^{6}$ Il y a aussi la vacance d'un emploi pour un an maximum, le remplacement de titulaires...
} 


\section{Un positionnement organisationnel spécifique}

En raison de leur jeunesse, les services fonctionnels transversaux ${ }^{7}$ de communication sont privés d'une légitimité historique d'antériorité, à la différence des directions opérationnelles sectorielles. Cet état de fait traduit la prise en compte d'enjeux sociétaux ${ }^{8}$ intégrés assez récemment par les édiles, de sorte que l'institutionnalisation des services de communication est toujours en consolidation.

En tant que services administratifs de l'État, ils concernent tous les ministères selon trois phases formalisées par Romain Laufer et Alain Burlaud (1980, pp. 160-166). La première réside dans le poste occupé par une personne du cabinet en charge des relations entre le public et le ministère, aidée d'une équipe de presse, puis on passe à un bureau officiel ; les relations avec les directions dépendant surtout des personnes et peu de l'organisation. Enfin, intervient la création d'un service ou d'une direction traitant des relations publiques avec des correspondants autonomes dans chaque direction. Ces différentes organisations produisent essentiellement des revues et des journaux, des écrits informatifs (brochures, ouvrages), de l'affichage et aujourd'hui mobilisent les technologies d'internet.

De tels services au niveau des collectivités locales remontent pour les plus anciens aux années 60 , avec les premiers bulletins municipaux à diffusion et périodicité faibles. Les années 70 sont celles de la constitution des premiers services de communication de certaines grandes villes 9 . Puis dans les années 80 , de façon quasi généralisée au-dessus de 40000 habitants, on rencontre largement des services de communication placés sous l'autorité du maire ou du directeur du cabinet et dirigés par des professionnels.

Ces évolutions organisationnelles profitent aux fonctions de communication. Elles manifestent une modi-

\footnotetext{
${ }^{7}$ D'autres services fonctionnels à forte technicité peuvent être dans des configurations proches, comme ceux des formateurs (Bessières, Grima, 1999), les services informatiques...

8 Thèmes de la société de communication et de l'information caractérisant la société post-industrielle par sa généralisation dans tous les champs sociaux

9 Toutefois, des exemples sont antérieurs comme Bordeaux (1966), Nice (1967), Lille, Toulouse, Metz.
}

fication de la représentation de l'État, à la recherche d'une légitimation davantage fondée sur l'action par rapport à des objectifs que sur celle classique du droit mesurant l'efficacité par conformité à la réglementation (Laufer, Burlaud, 1980, p. 36, p. 115).

L'existence de services fonctionnels est censée améliorer l'efficience globale et constitue un champ de compétences précis. Luttant contre la compartimentation administrative, ils veillent à organiser une certaine cohérence entre les composantes opérationnelles. Paradoxalement, ce travail englobant d'unification rend leur acceptation difficile, mais celle-ci s'opère progressivement entre les années 80 et 90 au niveau des administrations décentralisées (Bessières 1998, pp. 170-243).

Des responsables de communication départementaux $^{10}$ attestent d'une différence de culture professionnelle : "La hiérarchie administrative n'est pas du tout imprégnée de communication, parce que les services (opérationnels, ndlr) ne voient pas pourquoi il faut faire savoir alors qu'ils font leurs missions, avec les commissions et les élus, et qu'ils ont des partenaires extérieurs connus, ce qui les conforte. Cela tient à des problèmes de structure et à un esprit. Toutes les administrations, les collectivités territoriales fonctionnement comme ça». "Le fait d'être dans une structure administrative, c'est pour que la communication soit vécue dans la maison comme une activité à part entière et en même temps le rattachement politique montre le caractère stratégique de la fonction ». Notre enquête sur les responsables de communication franciliens montre que la majorité des services de communication sont une composante du cabinet, ou bien ont des liens forts avec lui dans l'organisation des décisions de communication (Bessières 1998, p. 193, pp. 225-226).

La reconnaissance des fonctions récentes de communication met en jeu différentes sources de légitimité, alors même que leur rôle dans le fonctionnement des organisations publiques est plus fortement questionné aujourd'hui par la logique de performance recherchée par la LOLF ${ }^{11}$. Ces fonctions récentes

\footnotetext{
${ }^{10}$ Entretiens de l'auteur, 1996 et 1997.

${ }^{11}$ Loi d'organisation relative aux lois de finance, $1{ }^{\text {er }}$ août 2001.
} 
tiennent aux dirigeants, à l'action dans l'organisation, à des reconnaissances extérieures.

\section{La prééminence des contractuels}

La jeunesse des fonctions de communication se révèle dans le statut professionnel. Il est marqué par une certaine fragilité des positions acquises, liée au statut de contractuel dont la durée dépend du pouvoir politique. La communication est souvent perçue comme non directement productive, rendant son évaluation difficile. Elle repose toujours sur une décision des dirigeants politiques, faute d'obligation juridique pour la mise en place de tels services (Bessières 1998, pp. 64-65). Au sein des collectivités locales, les trois quarts des services de communication sont rattachés aux plus hauts sommets hiérarchiques, directement auprès de l'exécutif ou via son cabinet. Certains estiment que 50 à $60 \%$ des responsables et directeurs de la communication ne sont pas fonctionnaires (Douay, 2008, p. 310). On peut trouver davantage de fonctionnaires dans des postes moins stratégiques.

Les caractéristiques globales de la population des communicateurs publics ne peuvent être déterminées avec exactitude, tant en matière d'effectifs réels que de statut contractuel ou fonctionnaire. Nous n'avons pas trouvé de telles statistiques exhaustives et fiables au niveau national. Par ailleurs, une population de professionnels, comme celle des communicateurs publics, ne se prête pas à l'utilisation d'une méthodologie de sondage faute d'une population mère suffisamment importante. Nous ne pouvons donc qu'en avoir des approximations au travers d'enquêtes quantitatives. Elles démontrent toutes une prééminence des contractuels.

Les responsables de la communication sont majoritairement contractuels, des concours spécifiques à cette fonction semblent pour l'heure exclus, les compétences requises sont rares (Crozier 1987, p. 288). Pourtant, certains choisissent de passer des concours de recrutement de la fonction publique territoriale, deux dans notre corpus $^{12}$. Ils pointent l'avantage de la sécurité d'emploi associée au statut de fonctionnaire, mais également deux travers. Un

12 Entretiens de l'auteur effectués en 1995 et 2007. premier réside dans le fait de ne pas être assuré d'intégrer un poste de communicateur public, c'est une sorte d'épée de Damoclès pesant sur eux : leur développement de carrière peut passer par d'autres postes administratifs classiques, leur poste de responsable comportant toujours un terme. Un second correspond à la rémunération modeste des grilles indiciaires de la fonction publique, qui implique pour des responsables d'obtenir des primes compensatrices pour être en phase avec leur poste de directeur de la communication: "Je suis un titulaire atypique. Je n'ai pas le salaire correspondant à ma position. Il $y$ a donc des indemnités compensatrices qui posent des problèmes de retraite par ailleurs $»^{13}$. La perte de ce poste entraine de facto celle de cette rémunération substantielle.

En outre, la faiblesse de la définition des modalités $\mathrm{d}^{\prime}$ exercice du métier, la jeunesse des formations diplômantes spécifiques concourent à renforcer le pouvoir de l'élu dans la relation salariale. Ceci a été confirmé par la jurisprudence du Conseil d'État qui admet des contractuels spécialisés dans les relations publiques participant à l'exécution d'un service public administratif d'une collectivité locale ${ }^{14}$.

Aussi, leur situation, difficile à normaliser, les prive d'une possibilité d'évolution de carrière au sein de la même institution. Les préfets exerçant un contrôle de légalité sur les contrats publics, la mobilité est essentiellement externe ${ }^{15}$.

Cependant, pour certains directeurs de la communication, le statut de contractuel est intéressant en termes de liberté et de salaire. "Pourquoi rester contractuel? Parce que c'est un espace de liberté et de meilleure rémunération. Quant à devenir fonctionnaire : à voir, si une VAE (validation des acquis de l'expérience, ndlr) existe pour une intégration d'office au grade de directeur territorial ou d'administrateur. Mais pas de concours, non merci. La VAE existe bien pour des diplomes universitaires, pourquoi pas pour des concours de la fonction

\footnotetext{
13 Entretien d'un responsable de communication départemental francilien en 1995.

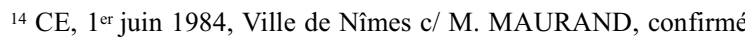
notamment par une autre jurisprudence CE, 23 octobre 1992, M. LALONDE.

15 Il existe des exemples de parcours de carrières importantes développées de cette façon.
} 


\section{Encadré 3}

\section{Enquêtes quantitatives auprès de responsables de communication locaux}

Les caractéristiques sociologiques de la population des communicateurs sont globalement difficiles à préciser, aussi des enquêtes quantitatives peuvent aboutir à des chiffrages notablement différents ; on note l'importance des contractuels, de la féminisation, un niveau de formation élevé :

- Enquête "Le directeur de la communication locale et Internet dans les collectivités : quelles influences sur le métier? », association communication publique, janvier 2006.

Méthodologie: questionnaire administré en ligne, 20 octobre-25 novembre 2005.

Répondants : 162

Statut de contractuel dominant (67\%), jusqu'à $93 \%$ pour les conseils généraux.

Niveau de formation : $87 \%$ ont au moins un bac +4 (dont $33 \%$ un bac +5 ). $56 \%$ ont une formation supérieure en communication soit en cursus unique, soit en formation complémentaire.

Féminisation: les hommes paraissent majoritaires dans cette enquête (56\%), jusqu'à 71 \% dans les régions, contre $30 \%$ de femmes dans les départements et $62 \%$ dans les mairies.

Source : blog.communication-publique.fr/ - 35k.

- Enquête "métiers 2005 Magazines Publics - Territoires et communication », 2005.

Méthodologie : questionnaire administré par courrier du 11 février au 11 mars 2005.

Répondants : 484.

Statut de contractuel le plus fréquent (44\%), les titulaires de la fonction publique représentent $37,5 \%$ des répondants (26\% par concours, $11 \%$ par intégration).

Niveau de formation : $36,5 \%$ ont un bac +4 et $32 \%$ un bac +5 ou plus $174 \%$ des responsables de la communication ont au moins un bac +4 ). $53 \%$ des directeurs de la communication ont une formation en communication (60\% des moins de 35 ans, contre $40 \%$ des plus de 45 ans).

Féminisation en progression parmi les répondants : $57,2 \%$ (contre 48,2 \% en 2002). Le taux dépasse $60 \%$ chez les moins de 35 ans et les villes les plus petites (-30 000 habitants).

Source : www. public-s.fr/carrieres/pdf/publics-carriere-61.pdf.

publique ?. $»^{16}$ On voit bien que les avantages sont essentiellement financiers. En effet, les contrats d'agents publics ne sont pas cadrés aussi fortement que les modalités de rémunération régulées par les grilles indiciaires. La perspective d'un concours après avoir développé un parcours professionnel de communicateur public semble rejetée. Tout se passe comme si le concours apparaissait trop académique et opposé en cela à l'exercice de pratiques professionnelles spécialisées.

Toutefois, récemment, la loi $\mathrm{n}^{\circ} 2005-843 \mathrm{du}$ 26 juillet $2005^{17}$ instaure un nouveau contrat à durée

${ }^{16}$ Entretien de l'auteur auprès d'un directeur de communication d'une importante ville de province, novembre 2008.

${ }^{17}$ La loi du 26 juillet 2005, en transposant le droit communautaire à la fonction publique, crée un nouveau statut de contractuel à .../... indéterminée " Fonction publique », assorti de conditions particulières. Il ne peut être proposé, sans obligation impérative, qu'au terme de six ans, après des contrats à durée déterminée de trois ans renouvelables une fois. Si cette mesure législative peut apparaitre comme un moyen de lutte contre la précarité, elle n'offre pas foncièrement de progression de carrière à l'égal des fonctionnaires titulaires. Ils resteront dans le poste et la fonction qu'ils occupent. Pour bénéficier de progression de carrière, les concours de recrutement des trois fonctions publiques

.../... durée indéterminée sur des emplois permanents. Peuvent en bénéficier tous les contractuels embauchés sous le régime de cette loi. Les autres contractuels, s'ils correspondent aux cas suivants : avoir au moins 50 ans, ou être en fonction ou bénéficier d'un congé au titre de la protection sociale des agents non titulaires, ou justifier d'une durée de services effectifs au moins égale à six ans effectifs au cours des huit dernières années. 
restent la norme. Ceci contraint les contractuels à se référer à une professionnalisation alternative.

Devant ces spécificités, pour pérenniser leur champ d'activité, les acteurs visent à s'instituer en groupe social avec des stratégies interactionnistes de représentation.

\section{L'AFFIRMATION DISTINCTIVE PAR INTERACTIONNISME PROFESSIONNEL}

L'approche interactionniste de la professionnalisation dévoile les actions des groupes sociaux pour se faire reconnaître vis-à-vis d'autres acteurs ou groupes (Dubar, Tripier, 2005, p. 90). Jusqu'aux années 90, les communicateurs publics, en particulier dans les collectivités locales, ont principalement cherché à exister d'abord au sein de leurs administrations par leur maitrise des outils et supports de communication. Leur légitimité à l'intérieur de leurs organisations et à l'extérieur (agences et journalistes) est instrumentale. En ce sens, l'enjeu pour ces acteurs est d'obtenir une reconnaissance par le biais de la légitimité de l'instrument communicationnel, c'est-à-dire par une légitimité professionnelle. Dans cette optique, les services fonctionnels de communication publique peuvent adopter une logique de «client-fournisseur » interne en agissant comme des prestataires de service auprès $\mathrm{du}$ pouvoir politique exécutif et de l'organisation administrative (Bessières-Grima 1999, pp. 33-34).

Ainsi en suivant Jacques Walter (1995, p. 12), on peut dire qu'ils utilisent le "répertoire mobilisable par un individu, ou un groupe, pour construire ou manifester sa compétence, en particulier sous contrainte de justification ». L'enjeu de la reconnaissance vise à déterminer « un modèle professionnel » de façon à consolider une place dans le champ social de ceux qui s'y reconnaissent.

Plus largement, construire une représentation globale du groupe est un moment classique de la constitution des groupes professionnels, c'est affirmer une définition. "En effet, un modèle est le produit d'expériences, d'observations, de réflexions sur le réel... Bref, il est un mode d'organisation de la réalité, qui oriente les représentations et les conduites sociales » (Walter 1995, p. 13). "Le groupe doit se faire représenter en se donnant des instances officielles et des porte-parole habilités à parler et à agir en son nom » (Boltanski 1982, p. 58). Ces instances fonctionnent comme des supports d'objectivation en donnant à voir comme une réalité tangible le champ des professionnels qu'elles promeuvent. Ces visions unificatrices s'inscrivent dans une approche interactionniste, portées par des associations, des salons, des codes déontologiques.

\section{Des représentations pour le groupe}

Les associations professionnelles ont pour fonction de promouvoir la reconnaissance sociale. Elles assurent "une garantie de l'existence et de l'unité dans la réalité sociale des professions, dans la mesure où elles sont à l'origine, sinon de la production, du moins de la systématisation et de la diffusion du stéréotype de la profession" (Chapoulie 1973, pp. 86-114).

L'association «Communication Publique» est de loin la plus importante dans le champ professionnel ${ }^{18}$. Constituée en 1989, selon son fondateur, elle regroupe principalement trois catégories d'institutions publiques : les administrations étatiques, les établissements publics, les collectivités locales. Quelques organismes relais ${ }^{19}$ regroupant des décideurs sont cooptés à des fins de confortement dans la sphère administrative. Elle compte 170 membres recensés dans le siège parisien. À l'instar de la plupart des autres associations professionnelles, elle constitue un lieu d'échanges, d'apprentissage ${ }^{20}$, d'études et de lobbying.

Son président fondateur, dans un entretien ${ }^{21}$, livre ses ambitions de légitimation : «Nous sommes une association de service public et nous avons été reconnus comme un lieu de passage. Nous sommes donc présents avec un grand stand d'accueil au Top Com, au Cap' Com, à l'Université de la communication

\footnotetext{
${ }_{18}$ On pourrait citer des associations pour la communication locale (Fourdin 1994).

${ }^{19}$ Service d'information du gouvernement pour l'État, l'Association des Maires de France, ou l'Association des Présidents de conseil général pour les collectivités locales.

${ }^{20}$ Elle est ouverte aux communicants publics et fermée aux agences.

${ }^{21}$ Entretien réalisé par l'auteur.
} 
d'Hourtin, comme partenaire animateur, ce qui permet d'être une vitrine des communicateurs $d u$ service public. » Il mentionne également un objectif d'études quantitatives et qualitatives comme un moyen « de promouvoir la fonction communication dans les services publics avec des études auprès des décideurs pour savoir ce qu'ils attendent des responsables de la communication. La presse est intéressée et en fait état $»$. De telles associations visent à promouvoir la profession et à constituer des organes de liaison entre praticiens.

Les prix professionnels inscrivent la profession dans un registre d'exemplarité récompensé par des pairs. Ils mettent en scène un modèle professionnel dans sa variété, la plupart des praticiens étant représentés. En ce sens, ils contribuent à forger une représentation. Ainsi, l'objectif du Cap' Com « vis-à-vis du consommateur est d'avoir des rencontres professionnelles avec une réflexion de qualité pour avoir de la distanciation et cerner les tendances d'un métier ${ }^{22}$. Depuis les années 90, l'association «Communication publique », son président ou certains de ses membres sont sollicités pour de nombreux prix professionnels de communication publique (campagnes, audiovisuels, mémoires de Master...).

Aussi les prix profitent collectivement à toute « la profession » dans son ensemble. L'objectif des organisateurs de ces manifestations professionnelles est de renforcer le rôle de communicateur au sein du secteur public. Le temps contribue à la consolidation d'un modèle professionnel en même temps que les salons et prix.

Ces manifestations sont des vecteurs d'objectivation et d'officialisation parce qu'elles rendent visibles une nomination publique pour le groupe lui-même et vis-à-vis d'autres groupes. Les salons et les prix jouent un rôle à cet égard. L'effet de réalité de ces opérations symboliques a été bien décrit par Luc Boltanski (1982, p. 57) : "Pour qu'un groupe apparaisse sur le tissu des relations sociales, il faut que soit forgé son concept et que soit institué son nom.» L'affirmation de ses caractéristiques vis-à-vis de luimême et des autres doit " donner des accentuations dramaturgiques de ses traits pertinents, sorte de

22 Entretien réalisé par l'auteur. stylisation qui contribue à la formation de la croyance collective sans laquelle le groupe n'a pas droit à la reconnaissance sociale ».

\section{Des propositions d'encadrement du métier}

La profession suppose une forme d'encadrement du métier évacuant le flou ${ }^{23}$. "La professionnalisation est un processus selon lequel un corps de métier tend à s'organiser sur le modèle des professions établies, c'est-à-dire, métiers qui ont développé un ensemble de caractéristiques spécifiques; monopole d'exercice de certaines fonctions, contrôle des praticiens par leurs pairs » (Chapoulie 1973, p. 89). En conséquence, un code de déontologie est symboliquement important dans l'affirmation d'une profession, même non établie, à l'exemple de celui de la publicité qui sert de référence au Conseil de la Concurrence ${ }^{24}$.

Le code déontologique organise une forme de reconnaissance sociale du groupe, de son expertise et de sa zone de pouvoir, en établissant des normes professionnelles, en rationalisant des pratiques, en proposant une identité (Walter 1995, pp. 20-22). L'association «Communication publique » a initié, en 1997, une réflexion déontologique aboutissant à une charte en 1998. L'exercice est symbolique d'une vision que des acteurs tentent d'imposer. Cette affirmation interactionniste du groupe est clairement affirmée par le secrétaire général de cette association (Douay, 2008, pp. 308-311).

L'association professionnelle délivre un discours produit et répété dans différents écrits et réunions. Des valeurs et des normes de reconnaissance sont ainsi diffusées. Son président fondateur évoque, en particulier, une communication d'utilité publique pour faire partager les décisions d'intérêt général, l'accueil et la relation au public en tant que composantes du service public offert. Ou encore que la communication s'oppose à la promotion en permettant échange et partage d'information. Des valeurs sont ainsi proclamées et proposées pour références identitaires, à destination des membres du réseau professionnel en particulier. L'association atteste la

${ }^{23}$ C'est une spécialisation professionnelle au sens de Dubar et Tripier (2005).

${ }^{24}$ Organisme de contrôle. 
spécificité du groupe par ses activités et son existence même. Ce groupement professionnel développe notamment un discours à destination des pouvoirs publics, avec une dimension de lobbying (Bessières, Ollivier-Yaniv., 1997, pp. 19-31) pour institutionnaliser les communicateurs publics en invitant dans un colloque nombre de décideurs politiques et en montrant de nouvelles compétences possibles pour les services de communication.

Au travers de ces représentations exemplaires proposées au groupe professionnel des communicateurs publics, on perçoit bien qu'il s'agit d'une lutte destinée à forger des représentations performatives. La recherche de consolidation est patente pour les professionnels de la communication publique.

Ces opérations de construction de valeurs et de représentations propres contribuent à définir une identité professionnelle spécifique au travers de dynamiques de reconnaissances sociales de certaines activités (Dubar, Tripier, 2005, p. 84). Cette identité repose sur un ensemble de signes ou d'emblèmes qui permettent de regrouper des professionnels (logos, discours, valeurs et modèles proclamés). Elle participe d'un processus d'institutionnalisation par la production de référents pour catégoriser et donc définir le groupe, offerts à ses membres mais aussi à l'extérieur.

Pourtant, si la construction sociale du groupe professionnel des communicateurs publics est indubitablement à l'œuvre, elle est problématique dans la mesure où elle repose sur un professionnalisme du flou. Le processus de professionnalisation des activités de communication publique participe à la construction de «professionnels» détenteurs d'un "pouvoir de l'expertise », le pouvoir politique étant corrélativement plus ou moins fortement dessaisi d'une partie de son activité communicationnelle (Neveu 2006, p. 101). Toutefois, l'appellation «communication publique » regroupe une grande diversité de fonctions effectives, de profils professionnels (chargé de communication, de relation publique et presse, journaliste...), de diplômes (droit, science politique, communication...) si bien que l'on ne trouve guère d'enjeux communs entre tous les communicateurs en dehors d'une quête constante de reconnaissance. Dès lors, les communicateurs publics sont un groupe imprécis et flou cherchant à obtenir une reconnais- sance en se distinguant. Isabelle Pailliart (1993, p. 99) a décrit cette spécificité du groupe par rapport à l'ensemble du personnel des collectivités publiques. Le flou des activités de communication (variabilité des périmètres entre conseils, gestion de prestataires, relations publiques et presse, événementiel, rédaction de discours, magazine, plaquettes, site web...) permet de dissimuler la diversité de la profession et des professionnels, tout en permettant une représentation d'unité pour imposer ce nouveau groupe professionnel en construction. On mesure ainsi l'intérêt du groupe pour la reconnaissance étatique et publique complétant cette dynamique.

\section{UNE RECONNAISSANCE ÉTATIQUE PARTIELLE MAIS INSTITUANTE}

L'État français représente le cadre d'exercice des communicateurs publics; c'est aussi un vecteur essentiel d'institutionnalisation de leur groupe professionnel par son action de régulation (Dubar, Tripier, 2005).

\section{L'action étatique structurante de la législation et de l'Université}

La division du travail aboutit à des rôles professionnels plus nombreux et spécialisés et dont l'accès est lié à une formation spécifique. L'émergence de filières de formation contribue à faire surgir la question de l'identité corporatiste qui donnera unité et sens à ce qui n'est au départ qu'une agrégation abstraite de rôles professionnels.

Ce processus se retrouve dans le champ de la communication publique. Le capital culturel pour exercer la profession s'élève progressivement, ce que traduit l'accroissement des formations en communication orientées vers le secteur public ${ }^{25}$. Luc Boltanski

\footnotetext{
${ }^{25}$ De façon non exhaustive, on peut citer, au niveau bac +5 , des pionniers à la fin des années 70 à Paris I : Diplôme d'études supérieures spécialisées «Communication politique et sociale » qui perdure en master. Durant les années 80 se sont développées des formations plus spécialisées (DESS « Politique et animation locale » Paris I), puis durant les années 90, en région parisienne (Celsa, Paris 12, Versailles Saint-Quentin en Yvelines), et de nouvelles formations tout récemment dans les Instituts d'études politiques (Bordeaux, Lille).
} 
(1982, p. 48-49, pp. 191-193) a montré l'importance du développement des formations qui assurent une production de nouveaux professionnels pour la constitution d'un groupe professionnel. Celui-ci peut se faire reconnaître comme une profession en se dotant progressivement d'attributs fonctionnels, comme des formations scientifiques spécialisées, avant de pouvoir exercer. La reconnaissance universitaire permettant d'acquérir, en particulier, des connaissances académiques (critère de performance) qui cessent d'être empiriques (apprentissage) (Dubar, Tripier 2005, pp. 83-84). L'intérêt porté par des membres des institutions universitaires, au travers de recherches scientifiques et d'enseignements, contribue à définir le champ des savoirs techniques et conceptuels de la communication publique, en l'officialisant et en le développant. Aussi, l'université agit comme une puissante instance de légitimation par la pérennité des diplômes, notamment au niveau bac +5 , qu'elle produit dans ce champ.

Le droit public français n'offre pas de définition positive de la communication publique, mais une reconnaissance implicite ${ }^{26}$, limitative, de certaines pratiques. Les possibilités de communication avant les élections sont limitées en vertu de la loi du 15 janvier 1990. Ainsi sont interdites au moins six mois avant un scrutin national, la personnalisation de la communication, la promotion publicitaire des réalisations ou de la gestion de nouvelles actions de communication. Les restrictions légales sont de nature à renforcer la légitimité des communicateurs institutionnels. En effet, elles contribuent à éliminer les communications politiques ouvertement personnalisées des élus au profit d'une communication d'apparence publique pérenne (Bessières 1998, pp. 326-417). Ces prescriptions légales sont un puissant facteur de renforcement du groupe des communicateurs publics parce qu'elles sont intégrées dans la pratique des organisations (Duran 1992, p. 6). Leurs applications concrètes relèvent de l'expertise des professionnels de la communication, dont la présence est rendue plus nécessaire en raison des risques de remise en cause des élections en cas de manquements. Plus largement, l'activité communicationnelle

26 À la différence de l'Italie, seul pays européen doté d'une loi régissant le champ (loi du 7 juin 2000 n. 150). est régie par des normes impersonnelles juridiques qui la légitiment en définitive (Laufer, Paradeise 1982, pp. 42-43).

\section{L'officialisation par les répertoires de métiers publics}

Trois répertoires sont autant de façons de voir les communicateurs publics, ce qui est problématique on ne reconnaît pas exactement les mêmes fonctions d'une part, et un succès - c'est déjà un effort de clarification et de reconnaissance d'autre part. Cependant, par leur existence même, ces répertoires des métiers publics témoignent d'une reconnaissance des spécificités de la communication publique par rapport à celle des entreprises, avec des similitudes partielles dans les trois fonctions publiques.

Le Répertoire interministériel des métiers de l'État (RIME) de 2006 témoigne de l'intégration du groupe des communicateurs dans les organisations étatiques, à la suite des référentiels métiers des fonctions publiques territoriales et hospitalières. Il concerne les emplois titulaires et contractuels. Il s'inscrit dans l'air du temps des référentiels de compétences courants dans les entreprises et les organisations en identifiant des compétences dans le souci d'améliorer le recrutement. Il constitue le pendant du Répertoire opérationnel des métiers et des emplois (ROME) pour le secteur privé. À ce titre, il renforce la légitimation des communicateurs publics par cette référence interministérielle commune. Il peut conduire à faire évoluer la rigidité du statut des fonctionnaires ${ }^{27}$ recrutés sur concours, décrite par Crozier (1987, p. 288), en permettant une certaine normalisation de ces fonctions transversales. En tout état de cause, il contribue à solidifier le positionnement des acteurs.

Les emplois-référence (types d'emplois nécessaires à la réalisation de ces fonctions) ont été définis à partir des domaines fonctionnels ${ }^{28}$ (ensemble homogène de fonctions concourant à la même finalité), en concertation avec les ministères et les syndicats.

\footnotetext{
${ }^{27}$ La reconnaissance de la notion de métier est récente dans la fonction publique ; interministérielle, elle est censée favoriser la mobilité en valorisant les compétences et les qualifications plutôt que les seuls concours de recrutement, et favoriser la construction de parcours professionnels.

28 ... et non pas à partir de l'analyse des emplois ministériels.
} 
Tableau 1

Les activités communication dans les répertoires des métiers des trois fonctions publiques

\begin{tabular}{|c|c|c|}
\hline $\begin{array}{l}\text { Fonction publique d'État } \\
10 \text { fiches métier }\end{array}$ & $\begin{array}{c}\text { Fonction publique territoriale } \\
6 \text { fiches métier }\end{array}$ & $\begin{array}{c}\text { Fonction publique hospitalière } \\
7 \text { fiches métier }\end{array}$ \\
\hline $\begin{array}{l}\text { Directeur de la communication } \\
\text { Chargé de communication } \\
\text { Chargé de presse } \\
\text { Chargé de la communication événe- } \\
\text { mentielle } \\
\text { Responsable des campagnes } \\
\text { Responsable audiovisuel } \\
\text { Chef de projet multimédia } \\
\text { Chargé de publication } \\
\text { Graphiste } \\
\text { Chargé de promotion et de diffusion } \\
\text { commerciale }\end{array}$ & $\begin{array}{l}\text { Directeur de la communication } \\
\text { Chargé de communication } \\
\text { Chargé de publication } \\
\text { Photographe-Vidéaste } \\
\text { Chef de projet multimédia } \\
\text { Créateur de support graphique et } \\
\text { audiovisuel }\end{array}$ & $\begin{array}{l}\text { Attaché de presse } \\
\text { Chargé de communication } \\
\text { Cinéaste-Vidéaste } \\
\text { Maquettiste-infographiste } \\
\text { Photographe } \\
\text { Webmestre } \\
\text { Technicien audiovisuel }\end{array}$ \\
\hline
\end{tabular}

Sources : Répertoire interministériel des métiers de l'État, Répertoire des métiers de la fonction publique territoriale, Répertoire de la fonction publique hospitalière.

Un membre volontaire de la commission d'élaboration $^{29}$ pour la communication, interviewé $e^{30}$, témoigne de son souci de reconnaissance pour la profession, lorsqu'il y avait débat sur les emplois référents en communication publique de l'État : «On contestait le métier de communicant. Est-ce que c'est une formation longue? Sinon tous les agents peuvent le faire. » Il signale que le RIME consacre la communication en tant que métier ${ }^{31}$, mais entre proximité et ignorance. Proximité, parce que dans le modèle de fiche initial, on prenait l'exemple du chargé de communication que tout le monde était censé comprendre. Ignorance, en revanche, en insistant sur la communication, simple fonction d'appui, pas vraiment stratégique. Ce reproche se s'adresse pas directement en fait au RIME qui distingue trois groupes de domaines fonctionnels : les fonctions stratégiques (les actions d'une organisation particulière, d'une phase d'élaboration, de pilotage et d'évaluation des politiques publiques), les fonctions opérationnelles (mise en œuvre de l'action à destination directe d'un

\footnotetext{
${ }^{29}$ Un groupe de travail dédié composé de 15 membres réunissant des représentants d'administrations centrales, sur la base du volontariat, animé par un président. Des compromis entre les membres ont permis d'élaborer le RIME sur une base de consensus.

${ }^{30}$ Entretien réalisé par l'auteur auprès d'un haut fonctionnaire spécialisé dans la communication publique, octobre 2007.

31 Terme choisi, en dépit de ses acceptions théoriques variées, pour des motifs de valorisation.
}

destinataire), les fonctions d'appui (activité servant le fonctionnement de l'État). Cela signifie qu'il apparaît souhaitable pour l'interviewé d'insérer «la communication en amont des décisions afin de réduire le différentiel entre les attentes et les projets ». La critique porte sur la place de la communication comme simple fonction d'appui.

L'enquêté regrette que la communication soit la dernière famille de métiers du répertoire (le $23^{\text {e }}$ domaine fonctionnel). Pour lui, c'est un cadre à enrichir, il faudrait une autre fiche métier pour la communication de crise, même si elle n'est pas une activité à plein temps.

Certaines fonctions n'ont ainsi pas été retenues par la commission au titre $\mathrm{d}^{\prime}$ « emplois référence $»^{32} \mathrm{du}$ domaine :

- ceux faisant partie d'autres secteurs : les postes de conseillers pour la communication et la presse auprès des ministres («conseillers du gouvernement»), la fonction d'accueil (un domaine «service aux usagers » comprenant 7 métiers), les personnels des centres de documentation (gestionnaire de ressources

\footnotetext{
${ }^{32}$ L'emploi-référence décrit de façon synthétique les emplois de l'État. C'est un regroupement d'emplois types suffisamment proches pour pouvoir être exercés par les mêmes personnes, moyennant adaptation ou formation.
} 
documentaires et éditoriales de l'administration générale) ;

- ceux propres à la fonction communication, mais qui ne sont pas encore reconnus comme des emploisréférence parce qu'ils nécessitent une formation complémentaire d'un an: fonctions "études et veille» (intégrées à celle de responsable des campagnes), les agents dédiés à la passation des marchés publics de communication (acheteur public), les chargés de la communication interne, y compris au niveau territorial.

Néanmoins, le RIME est clairement un facteur d'institutionnalisation du groupe par la reconnaissance officielle de la professionnalisation des fonctions de communication qu'il énonce. Par la définition des savoir-faire et des connaissances nécessaires, il constitue un référentiel pour l'emploi public et les parcours professionnels, quels que soient le corps d'origine, le statut, le ministère d'affectation.

\section{Une identité professionnelle publique}

La communication publique reproduit la communication d'entreprise dans ses attributs symboliques (techniques communicationnelles de construction d'identité d'organisation, par projections de valeurs d'adhésion consensuelles, logos...), dans une dimension verticale qui relie l'institution à la population par l'entremise de la communication, en ne ménageant pas de grandes possibilités de dialogue. Cette communication identifie la logique institutionnelle de la prééminence de l'exécutif concentrant la plus grande part du pouvoir politique local qui résulte de la décentralisation ${ }^{33}$.

L'argument distinctif de la communication du service public est souvent mentionné par les professionnels dans différentes manifestations ${ }^{34}$. Un des représentants du groupe professionnel (Zémor, 2008) signale que des messages publicitaires appliqués dans le champ public peuvent aboutir à reprendre des thèmes similaires galvaudés et tombés dans la bana-

\footnotetext{
${ }^{33}$ Les conditions juridiques des concertations ne remettent pas en cause le système représentatif des exécutifs, puisque ces derniers en contrôlent le cadrage et ne sont pas juridiquement liés à leur résultat.

${ }^{34}$ Différents entretiens et observations de l'auteur.
}

lité (thèmes des technopôles, centres et carrefours de l'Europe...). Un journaliste spécialisé dans la communication locale, au cours d'un entretien, la différencie de la communication d'entreprise au motif qu'elles n'ont pas les mêmes besoins. " $L a$ communication locale n'est pas spécifique par les techniques utilisées - journal local... Les agences de communication leur ont vendu de la publicité pendant des années à tort. Il vaut mieux un travail de terrain, les relations publiques, les relations presse, les journaux locaux $»^{35}$. De sorte que la communication privée n'apparaît plus représentative et encore moins un modèle pour la communication publique. La communication publicitaire des années 80, souvent dénommée péjorativement "paillettes », est largement rejetée comme un contre-modèle qui constitue un stéréotype reconnu de bonne professionnalité. Au total, le caractère public apparaît comme une essence $^{36}$ de nature à définir la communication institutionnelle publique. De sorte que le contexte d'action étatique contribue à définir la spécificité des fonctions de communicateurs publics.

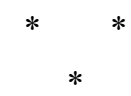

Les communicateurs publics ne peuvent pleinement intégrer la professionnalisation par concours, dominante dans le secteur public. Ceci contribue à considérer les agents contractuels en charge de communication publique comme «marginaux», même s'ils sont majoritaires dans le domaine. Aussi, collectivement, ils recherchent des stratégies alternatives de professionnalisation calquées sur celles du secteur privé. Ce processus de consolidation se renforce continuellement depuis les années 80. Il articule des éléments macrosociaux (droit, université, référentiels métier) d'une part, et microsociaux (déontologies, salon et prix, associations, acteurs des organisations publiques) d'autre part.

Cette dynamique d'affirmation de reconnaissance et de légitimation en groupe professionnel des communicateurs existe, même si les hommes, les outils sont

\footnotetext{
35 Entretien auprès d'un journaliste spécialisé.

36 En application du régime juridique, et du comportement propre au secteur public.
} 
perçus comme évolutifs et non stabilisés. Cette plasticité est un vecteur de pérennisation d'une activité progressivement institutionnalisée par l'extérieur et l'intérieur des institutions publiques. On assiste bel et bien à la construction d'une réalité qui tend à devenir objective au travers d'instruments, de services, de formations, d'associations professionnelles, de répertoires officiels, tangibles. Aussi, comme le souligne Pierre Bourdieu (1982, pp. 126-127), «instituer, assigner une essence, une compétence, c'est imposer un droit d'être qui est un devoir être (ou d'être). «[...] « Deviens ce que tu es, telle est la formule qui sous-tend la magie performative de tous les actes d'institution ». Le programme consiste à faire considérer une telle activité sociale comme allant de soi. Le temps est incontournable pour y parvenir.
Tout se passe comme s'il existait un impératif d'application des critères des professions; lesquels sont collectivement instrumentalisés dans une stratégie de renforcement des communicateurs publics. Ils en appliquent les règles, mais on mesure que la légitimation du groupe est une condition d'intégration pérenne dans une structure de travail fonctionnarisée, dans la mesure où elle est l'objet d'opération collective d'imposition et de reconnaissance. Les acteurs sont informés des modèles de groupes de communicants antérieurs («publicitaires », Neveu, 2006 ; « conseils en communication politique », Champagne, 1990, p. 30). Derrière l'imitation, c'est bien une légitimation professionnelle garante d'une institutionnalisation durable qui est recherchée dans une professionnalisation problématique et ambivalente.

\section{Bibliographie}

Bessières D. (1998), L'institutionnalisation de la communication locale - les cas des échelons décentralisés départementaux, régionaux, parisiens, franciliens, Doctorat de science politique, université Paris I Panthéon-Sorbonne.

Bessières D., Grima F. (1999), «Quelles légitimités pour les services fonctionnels de la communication et de la formation dans les collectivités locales?», Politiques et Management Public, $\mathrm{n}^{\circ}$ 4, décembre.

Bessières D., Ollivier-Yaniv C. (1997), « La communication départementale : constat d'une arlésienne ou processus de légitimation? », Quaderni, $\mathrm{n}^{\circ} 31$.

Boltanski L. (1982), Les cadres, la formation d'un rôle social, Paris, Éditions de Minuit, coll. « Le sens commun ».

Bongrand Ph., Laborier P. (2005), «L'entretien dans l'analyse des politiques publiques: un impensé méthodologique? », Revue française de science politique, vol. $55 \mathrm{n}^{\circ} 1$, février.
Bourdieu P. (1982), Ce que parler veut dire L'économie des échanges linguistiques, Paris, Fayard.

Champagne P. (1990), Faire l'opinion, Paris, Éditions de Minuit, coll. « Le sens commun ».

Chapoulie J.-M. (1973), « Sur l'analyse sociologique des groupes professionnels ", Revue Française de Sociologie, $_{2}$ vol. XIV, $\mathrm{n}^{\circ} 1$.

Crozier M. (1987), État moderne, État modeste, Paris, Fayard.

Douay P.-A. (2008), « Carrières et formation », in La communication publique en pratiques, sous la direction de Lemaire M. et Zemor P., La Documentation française.

Dubar C., Tripier P. (2005), Sociologie des professions, 2e éd., Armand Colin.

Duran P. (1992), «Piloter l'action publique, avec ou sans le droit», Revue Politique et Management Public, vol. 11, $\mathrm{n}^{\circ} 4$, décembre. 
Fourdin M. (1994), «La professionnalisation de la communication locale: un paradoxe?», Réseaux $\mathrm{n}^{\circ} 64$, mars-avril.

Gadéa Ch. (2003), Les cadres en France, Paris, Belin, coll. « Perspectives sociologiques ».

Laufer R., Burlaud A. (1980), Management public gestion et légitimité, Paris, Dalloz, Coll. «Dalloz Gestion Systèmes et Stratégies ».

Laufer R., Paradeise C. (1982), Le prince bureaucrate, Paris, Flammarion.
Pailliart I. (1993), Les territoires de la communication, Grenoble, PUG.

Thoenig J.-C. (1985), «L'analyse des politiques publiques ", in Grawitz M. et Lecas J., Traité de science politique, vol. IV, Paris, PUF.

Walter J. (1995), Directeur de communication - Les avatars d'un modèle professionnel, Paris, L'Harmattan, coll. « Logiques Sociales ».

Zemor P. (2008), La communication publique, Que sais-je?

Neveu E. (2006), Une société de communication?, Paris, Montchrétien, coll. « Clefs politique ».

\section{Résumé \\ La quête de professionnalisation des communicateurs publics : entre difficulté et stratégie \\ Dominique Bessières}

Les chargés de communication publics, souvent contractuels, ne peuvent pleinement intégrer le modèle dominant du fonctionnaire recruté par concours. Aussi, depuis les années 80, ces agents "marginaux» recherchent des stratégies alternatives de professionnalisation pour conforter leur position minoritaire. Cette quête de professionnalisation du groupe professionnel peut s'avérer problématique, même si collectivement et successivement, ces acteurs ont su mobiliser des ressources publiques, politiques macrosociales (droit, université, référentiels métier) et microsociales (déontologie, salons et prix, associations...) qui s'avèrent stratégiques.

Mots clés :

Professionnalisation, chargé des relations publiques

Journal of Economic Literature : J 44 